\title{
Recent MAGIC results on Galactic binaries
}

\section{E. Molina, ${ }^{a, *}$ A. López-Oramas, ${ }^{b}$ D. Hadasch ${ }^{c}$ and J. Hoang ${ }^{d, e}$ on behalf of the MAGIC Collaboration ${ }^{\dagger}$}

${ }^{a}$ Departament de Física Quàntica i Astrofísica, Institut de Ciències del Cosmos (ICCUB), Universitat de Barcelona (IEEC-UB),

Martí i Franquès 1, 08028 Barcelona, Spain

${ }^{b}$ Instituto de Astrofísica de Canarias and Dpto. de Astrofísica, Universidad de La Laguna, E-38200, La Laguna, Tenerife, Spain

${ }^{c}$ Institute for Cosmic Ray Research (ICRR), The University of Tokyo, Kashiwa, 277-8582 Chiba, Japan

${ }^{d}$ IPARCOS Institute and EMFTEL Department, Universidad Complutense de Madrid, E-28040 Madrid, Spain

${ }^{e}$ Department of Astronomy, University of California Berkeley, Berkeley CA 94720, USA

E-mail: emolina@fqa.ub.edu

X-ray and gamma-ray binaries are systems consisting of a compact object and normally a nondegenerate companion star. Most of these sources have been shown to emit radiation in a broad frequency range, from radio up to X-rays and sometimes gamma rays. We report on recent results in very high-energy gamma rays above $100 \mathrm{GeV}$ obtained by the MAGIC Collaboration for the Galactic X-ray binaries MAXI J1820+070 and 1A 0535+262, and the gamma-ray binary HESS J0632+057. Multiwavelength data at lower energies are also provided for a better contextualisation of the sources.

$37^{\text {th }}$ International Cosmic Ray Conference (ICRC 2021)

July 12 th - 23rd, 2021

Online - Berlin, Germany

\footnotetext{
${ }^{*}$ Presenter

${ }^{\dagger}$ a complete list of the MAGIC Collaboration authors can be found at the end of the proceedings
} 


\section{Introduction}

There are a number of binary systems in our Galaxy, typically consisting of a compact object and a non-degenerate companion star, that produce X-ray and gamma-ray emission. Depending on the energy at which their emission peaks, they are called either X-ray or gamma-ray binaries. Two main scenarios have been proposed to explain the observed radiation, one involving matter accretion and jet launching by the compact object (microquasar scenario), and another one in which the compact object is a pulsar that interacts with the star through their winds (pulsar wind scenario).

MAXI J1820+070 is a low-mass microquasar with a black hole that was discovered in Xrays on March 112018 (MJD 58188.8) by the MAXI instrument [1]. Soon after its discovery, MAXI J1820+070 reached an exceptionally high X-ray flux peaking at $\sim 4$ times that of the Crab Nebula in the $15-50 \mathrm{keV}$ range [2,3]. The distance to the source was set to $2.96 \pm 0.33 \mathrm{kpc}[4]$, and an orbital period of $16.4518 \pm 0.0002 \mathrm{~h}$ was found [5]. Regarding its X-ray state, MAXI J1820+070 followed the usual cycle for microquasars with a black hole in outburst [6], going from a hard spectral state (HS) to a soft state (SS), and back to the HS shortly before going into quiescence [3]. The bottom panel in Fig. 1 allows to visualise the X-ray state evolution of the source throughout the outburst.

HESS J0632+057 is a gamma-ray binary consisting of a compact object and a massive Be-type star. It was discovered in very high-energy (VHE) gamma-rays as an unidentified point-like source during H.E.S.S. observations of the Monoceros region [7], and since then it has been detected at different frequencies from radio to high-energy gamma-rays. The distance to the source was set to $1.1-1.7 \mathrm{kpc}$ [8], and the orbital period to $316.8_{-1.4}^{+2.6}$ days from X-ray data [9]. The pulsar-wind scenario has been proposed for HESS J0632+057 [10, 11], although the microquasar scenario cannot be ruled out.

1A $0535+262$ is a Be X-ray binary composed of a giant Be-type star and a pulsar. This source has undergone periodic X-ray flares every few years since its discovery in 1975 (see [12] and references therein). In November 2020, it displayed an especially bright X-ray outburst reported by Swift/BAT [13] and confirmed by MAXI [14]. This is the brightest X-ray event recorded from this source up to date, reaching a luminosity level of $12 \mathrm{Crab}$ in the $15-50 \mathrm{keV}$ band in 19 November 2020. 1A $0535+262$ was detected in radio for the first time during the 2020 outburst [15], indicating the presence of non-thermal emission mechanisms. The distance to the source is $2.1 \pm 0.2 \mathrm{kpc}$ [16], and it has an orbital period of $111 \pm 0.4$ days [17]. Super-critical accretion onto the pulsar during the outburst was reported by [18].

In these proceedings, we provide an overview of the latest results on Galactic binary systems obtained with the MAGIC telescopes at energies above $100 \mathrm{GeV}$. In particular, we present results on the three sources described above: MAXI J1820+070, HESS J0632+057 and 1A 0535+262. We put these observations into a multi-wavelength context and discuss their physical implications for the different observed sources. The structure of the proceedings is the following: Sect. 2 describes the observations performed. In Sect. 3, the results for each source are shown and a discussion is provided. Finally, a short summary is given in Sect. 4. 
Table 1: Summary of the observations of MAXI J1820+070 with the MAGIC telescopes, after data quality cuts. The effective observation time and zenith angle range are shown for each source state, as well as for the whole data set.

\begin{tabular}{ccc}
\hline \hline Source state & Time $[\mathrm{h}]$ & Zenith angle $[\mathrm{deg}]$ \\
\hline Hard State & 14.2 & $21-58$ \\
$\mathrm{HS} \rightarrow \mathrm{SS}$ & 4.9 & $21-48$ \\
$\mathrm{SS} \rightarrow \mathrm{HS}$ & 3.4 & $28-56$ \\
\hline TOTAL & 22.5 & $21-58$ \\
\hline
\end{tabular}

\section{Observations and data analysis with MAGIC}

MAGIC [19] is a stereoscopic system of two Imaging Atmospheric Cherenkov Telescopes located at the Roque de los Muchachos Observatory in La Palma, Spain $\left(29^{\circ} \mathrm{N}, 18^{\circ} \mathrm{W}, 2200 \mathrm{~m}\right.$ above sea level). Each telescope has a 17-m diameter mirror dish that collects and focuses light into its focal plane, where a fast photo-multiplier tube camera with a $3.5^{\circ}$ field of view is located. The analysis of the MAGIC data presented here was done following the standard procedure described in [20].

\subsection{MAXI J1820+070}

MAXI J1820+070 was observed with the MAGIC telescopes during the X-ray outburst from March to October 2018, covering the initial HS of the source as well as the state transitions. A total of $22.5 \mathrm{~h}$ of data survived the quality cuts, all of them taken under dark conditions. These observations were performed together with the H.E.S.S. and VERITAS Collaborations for an added total of $61 \mathrm{~h}$ of good-quality data. In these proceedings we only report on the MAGIC observations, for which a summary can be found in Table 1 . We refer the interested reader to an upcoming publication for the details of the joint observational campaign of MAGIC, H.E.S.S. and VERITAS.

\subsection{HESS J0632+057}

The gamma-ray binary HESS J0632+057 was observed with MAGIC between October 2010 and November 2017, accumulating a total of $68 \mathrm{~h}$ of data. Some of these data were already published in [21]. After quality cuts, a total of $57.4 \mathrm{~h}$ remain. The data were taken under dark and moderate-to-strong moonlight conditions, and with zenith angles between 38 and $67^{\circ}$. The presence of a bright night sky background for the moon observations increases their energy threshold with respect to dark observations from 147 to $251 \mathrm{GeV}$. The MAGIC observations of HESS J0632+057 are part of a larger joint campaign with H.E.S.S. and VERITAS.

\section{$2.3 \quad 1 \mathrm{~A} \quad 0535+262$}

1A 0535+262 was observed with MAGIC between November 17 and December 192020 , contemporaneously to the giant X-ray flare. The bulk of the MAGIC observations took place during the peak of X-ray emission around November 19 (MJD 59172). After the data quality selection, which accounts for non-optimal weather conditions, $8.2 \mathrm{~h}$ of observations remained for further analysis, all of them done under dark conditions and at zenith angles below $35^{\circ}$. 


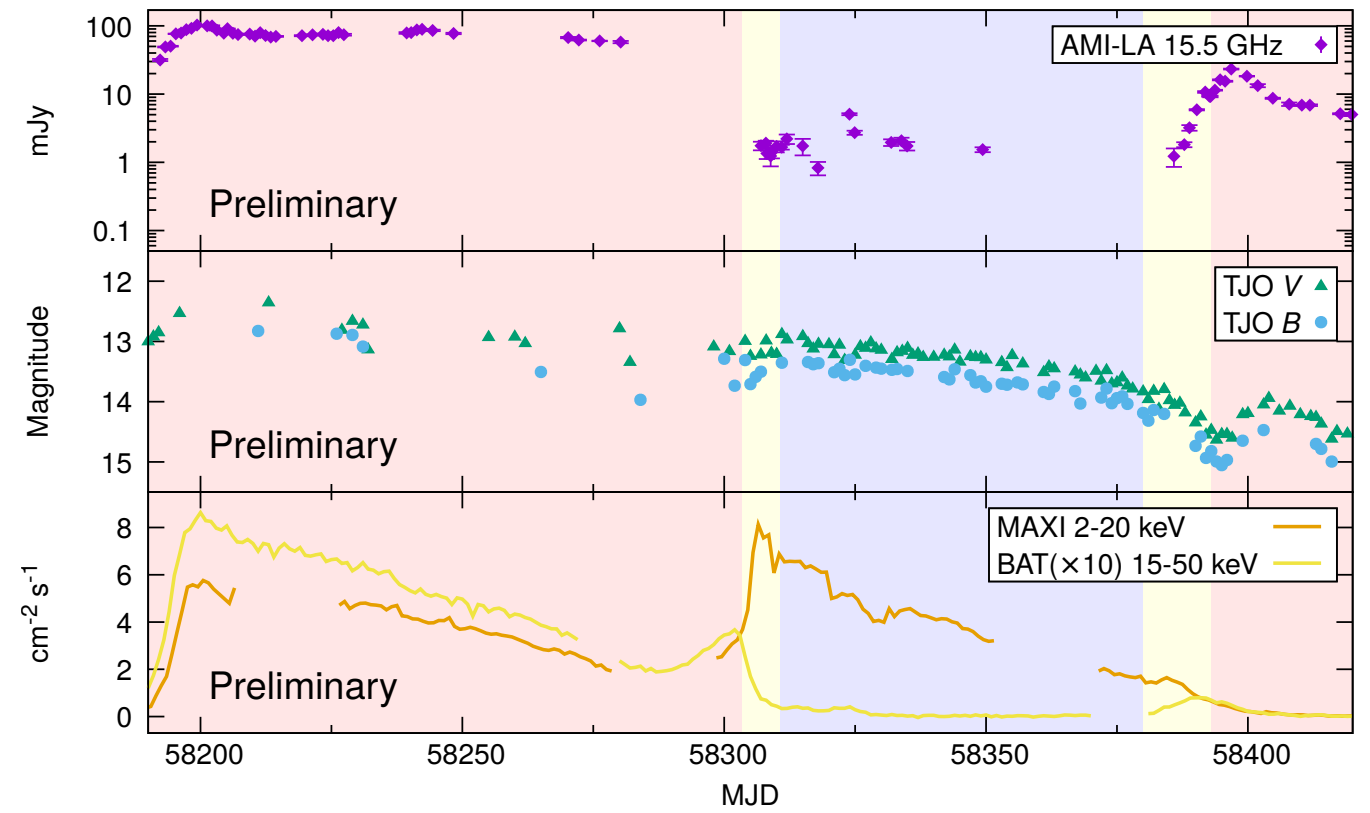

Figure 1: From top to bottom: Radio, optical and X-ray light curves of MAXI J1820+070 during its 2018 outburst. The shaded areas correspond to the HS (light red), the HS-SS and SS-HS transitions (yellow), and the SS (light blue). The units of the bottom panel are $\left[\mathrm{ph} \mathrm{cm}^{-2} \mathrm{~s}^{-1}\right.$ ] for MAXI/GSC, and [counts cm $\mathrm{cm}^{-1} \mathrm{~s}^{-1}$ for Swift/BAT. The latter fluxes are multiplied by 10 for a better visualisation.

\section{Results and discussion}

\subsection{MAXI J1820+070}

The MAGIC observations of the outburst of MAXI J1820+070 do not yield a significant detection of the source, and an integral flux upper limit (UL) above $200 \mathrm{GeV}$ of $2.2 \times 10^{-12} \mathrm{~cm}^{-2} \mathrm{~s}^{-1}$ is obtained for the full data set. This value is computed using a $95 \%$ confidence level and assuming a power-law spectrum with index -2.5 .

The light curve of MAXI J1820+070 during the outburst, as seen by different instruments, is shown in Fig.1. Radio data from AMI-LA is taken from [22], while optical data in the $V$ and $B$ filters from the Joan Oró Telescope (TJO; [23]) are taken from [24]. We also include public light curves from MAXI/GSC ${ }^{1}$ and Swift/BAT ${ }^{2}$. Without accounting for the radio emission of transient ejections launched during the HS-SS transition, which are dominant throughout the SS [22], the radio and hard X-ray fluxes have similar behaviours. They follow the standard picture of black-hole X-ray binaries, for which steady radio jets in the HS coexist with a hard X-ray emitting corona, both of them disappearing in the SS. These multiwavelength observations, together with the obtained VHE ULs, allow to set constraints on the size and position of a potential gamma-ray emitter in MAXI J1820+070. A detailed discussion on this issue will be given in a future joint publication of MAGIC, H.E.S.S. and VERITAS.

${ }^{1}$ http://maxi.riken.jp/star_data/J1820+071/J1820+071.html

2https://swift.gsfc.nasa.gov/results/transients/weak/MAXIJ1820p070 


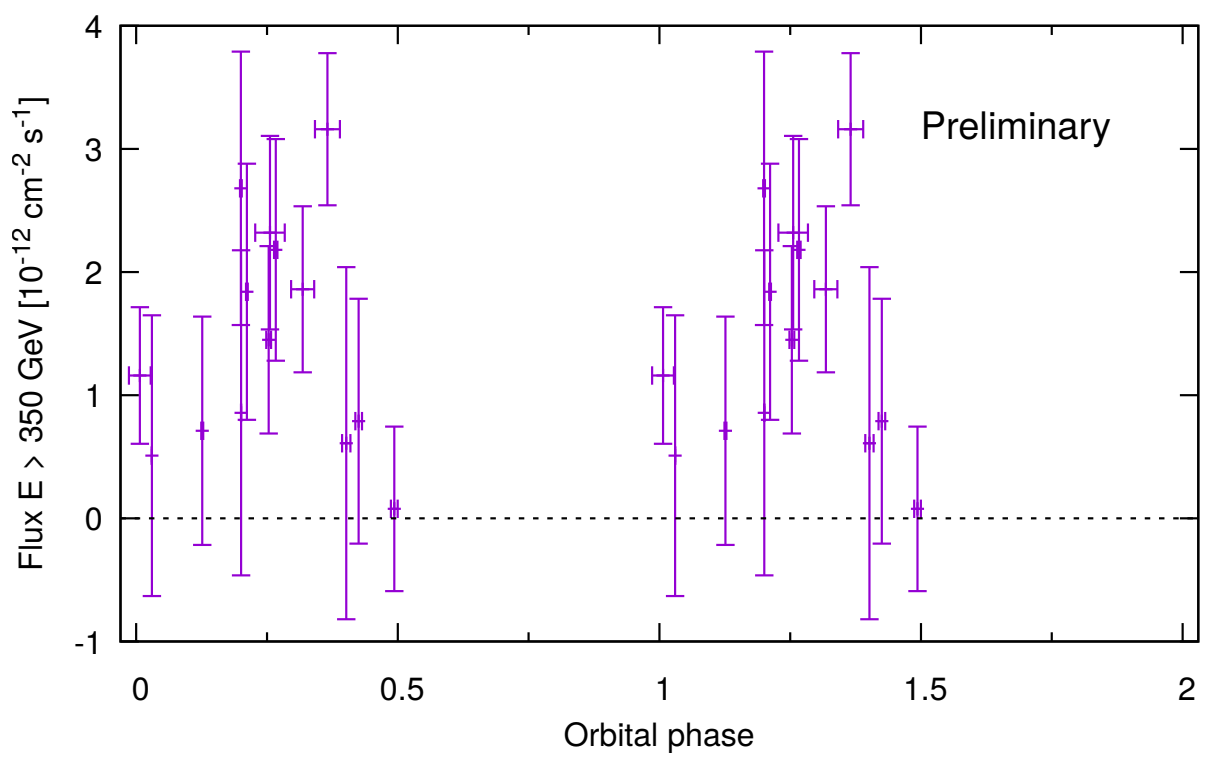

Figure 2: Phase-folded light curve of HESS J0632+057 assuming an orbital period of 316.8 days at energies above $350 \mathrm{GeV}$, as seen by MAGIC. Two orbits are shown for visualisation purposes.

\subsection{HESS J0632+057}

The long-term observations of HESS J0632+057 have allowed to better sample its VHE emission along the orbit. The VHE light curve obtained from MAGIC data above $350 \mathrm{GeV}$ is shown in Fig. 2, phase folded with an orbital period of 316.8 days. The observations were mainly done at orbital phases between 0 and 0.5. A maximum in the flux is found in the orbital range $0.2-0.4$, and a minimum is present at phases around 0.5 .

The results of the HESS J0632+057 analysis are part of a larger long-term observational campaign involving also the H.E.S.S. and VERITAS telescopes. This multi-year sampling of HESS J0632+057 allowed for the first-time determination of the orbital period from VHE data only, consistent with the previously determined X-ray period of 316.8 days [9]. A forthcoming publication will show the combined results of the three Collaborations, and will also include Xray and optical data. We refer to this upcoming joint publication for a detailed discussion of the observations and the interpretation of the results.

\subsection{A $0535+262$}

The analysis of the $8.2 \mathrm{~h}$ of observations of $1 \mathrm{~A} 0535+262$ does not reveal any significant VHE signal in the MAGIC data. The detection significance of the whole data set is $1.3 \sigma$ (computed following the usual Li\&Ma formula [25]). The integral flux UL at energies above $100 \mathrm{GeV}$ is $5.0 \times 10^{-12} \mathrm{~cm}^{-2} \mathrm{~s}^{-1}$ (with a $95 \%$ confidence level and an assumed power-law spectral index of -2.6). Fig. 3 shows the light curve of 1A 0535+262 in VHE gamma rays and X-rays, the latter extracted from public Swift/BAT ${ }^{3}$ and $\mathrm{MAXI}^{4}$ data. All the individual VHE flux points have a

3https://swift.gsfc.nasa.gov/results/transients/1A0535p262/

${ }^{4}$ http: //maxi.riken.jp/star_data/J0538+263/J0538+263.html 


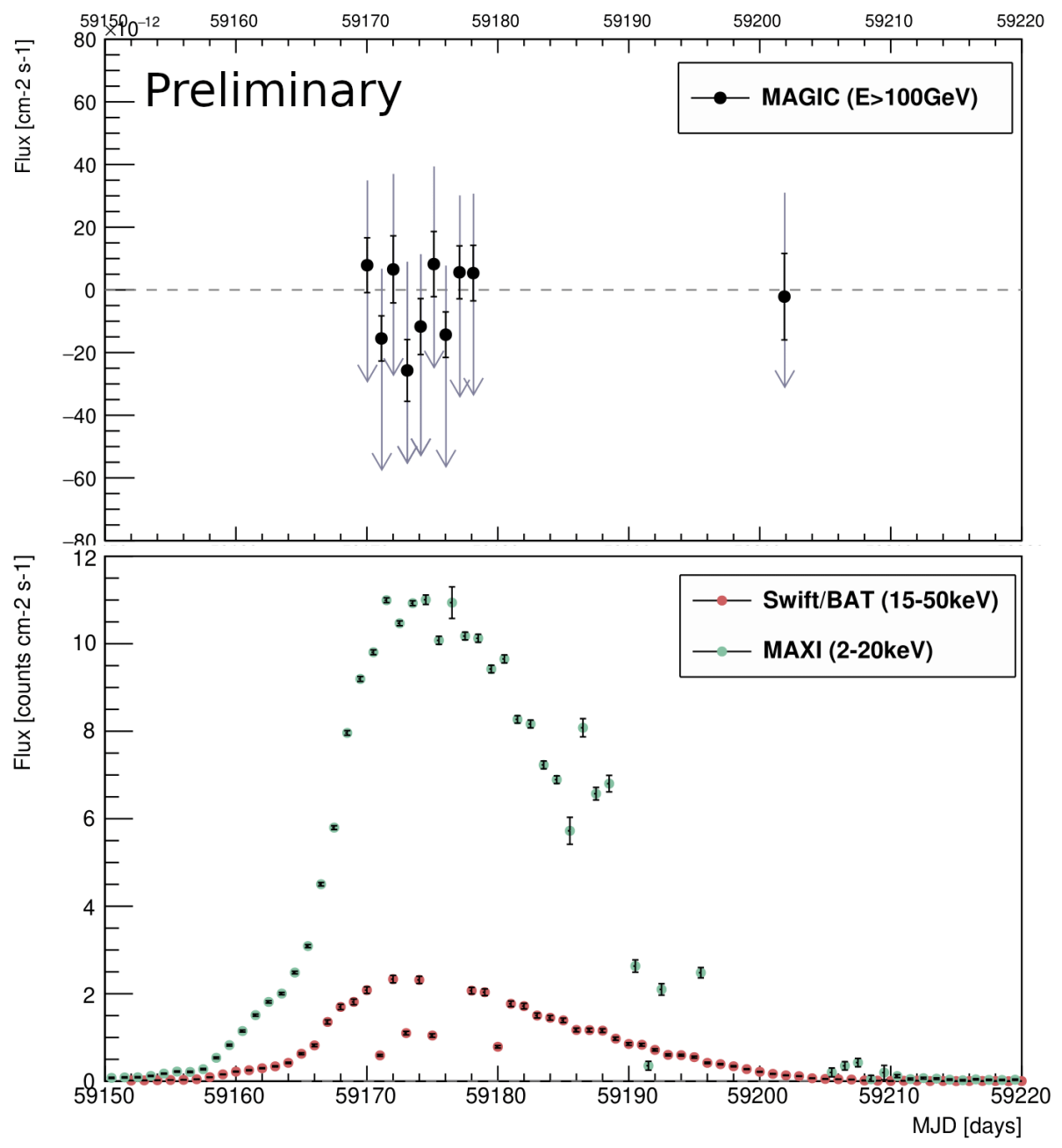

Figure 3: Top panel: VHE light curve of 1A $0535+262$ above $100 \mathrm{GeV}$ for the source outburst in 2020. The points show the computed fluxes, whereas the grey arrows represent the corresponding ULs at a $95 \%$ confidence level. Bottom panel: X-ray light curve of 1A 0535+262 as seen by Swift/BAT and MAXI in the same time period. Both panels use a daily time binning.

significance below $2.5 \sigma$, and the corresponding ULs are also shown.

\section{Summary}

We have reported on recent VHE gamma-ray obtained with the MAGIC telescopes for the Galactic binary sources MAXI J1820+070, HESS J0632+057 and 1A 0535+262. For the first two sources, upcoming joint publications with H.E.S.S. and VERITAS will provide more details on the results and the corresponding discussion. MAXI J1820+070 is not detected at VHE and flux ULs are computed, which together with the multiwavelength data from radio to X-rays allow to put constraints on a potential VHE emitter in the source. A clear orbital modulation is observed in 
HESS J0632+057 at VHE, and the combined MAGIC, H.E.S.S. and VERITAS observations of the source allowed to determine for the first time its orbital period from VHE data only. Finally, the brightest recorded X-ray flare from 1A 0535+262 has not come with detectable VHE emission, and integral flux ULs are reported.

\section{Acknowledgments}

We acknowledge the support from the agencies and organisations listed here:

https://magic.mpp.mpg.de/acknowledgments_ICRC2021

\section{References}

[1] T. Kawamuro, H. Negoro, T. Yoneyama, S. Ueno, H. Tomida, M. Ishikawa et al., MAXI/GSC detection of a probable new X-ray transient MAXI J1820+070, The Astronomer's Telegram 11399 (2018) 1.

[2] M. Del Santo and A. Segreto, The hard X-ray spectrum of MAXI J1820+070 observed by Swift/BAT, The Astronomer's Telegram 11427 (2018) 1.

[3] M. Shidatsu, S. Nakahira, K.L. Murata, R. Adachi, N. Kawai, Y. Ueda et al., X-Ray and Optical Monitoring of State Transitions in MAXI J1820+070, ApJ 874 (2019) 183 [1903.01686].

[4] P. Atri, J.C.A. Miller-Jones, A. Bahramian, R.M. Plotkin, A.T. Deller, P.G. Jonker et al., A radio parallax to the black hole X-ray binary MAXI J1820+070, MNRAS 493 (2020) L81 [1912 . 04525].

[5] M.A.P. Torres, J. Casares, F. Jiménez-Ibarra, T. Muñoz-Darias, M. Armas Padilla, P.G. Jonker et al., Dynamical Confirmation of a Black Hole in MAXI J1820+070, ApJL 882 (2019) L21 [1907. 00938 ].

[6] R. Fender and T. Muñoz-Darias, The Balance of Power: Accretion and Feedback in Stellar Mass Black Holes, in Astrophysical Black Holes, Lecture Notes in Physics, Volume 905. ISBN 978-3-319-19415-8. Springer International Publishing Switzerland, 2016, p. 65, vol. 905, p. 65, Springer International Publishing Switzerland (2016), DOI.

[7] F.A. Aharonian, A.G. Akhperjanian, A.R. Bazer-Bachi, B. Behera, M. Beilicke, W. Benbow et al., Discovery of a point-like very-high-energy $\gamma$-ray source in Monoceros, A\&A 469 (2007) L1 [0704.0171].

[8] C. Aragona, M.V. McSwain and M. De Becker, HD 259440: The Proposed Optical Counterpart of the $\gamma$-ray Binary HESS J0632+057, ApJ 724 (2010) 306 [1009. 2100].

[9] D. Malyshev, M. Chernyakova, A. Santangelo and G. Pühlhofer, Decade-long X-ray observations of HESS J0632+057, Astronomische Nachrichten 340 (2019) 465 [1711.05001].

[10] Y. Moritani, A.T. Okazaki, A.C. Carciofi, A. Imada, H. Akitaya, N. Ebisuda et al., Probing the Nature of the TeV $\gamma$-Ray Binary HESS J0632+057 by Monitoring Be Disk Variability, ApJL 804 (2015) L32 [1504.03067].

[11] M.V. Barkov and V. Bosch-Ramon, A hydrodynamics-informed, radiation model for HESS J0632 + 057 from radio to gamma-rays, MNRAS 479 (2018) 1320 [1806.05629].

[12] I. Caballero, P. Kretschmar, A. Santangelo, R. Staubert, D. Klochkov, A. Camero et al., A $0535+26$ in the August/September 2005 outburst observed by RXTE and INTEGRAL, A\&A 465 (2007) L21 [astro-ph/0702536]. 
[13] M. Mandal, S. Pal, M. Hazra et al., Swift/BAT detection of flaring activity from X-ray binary pulsar A 0535+262, The Astronomer's Telegram 14157 (2020) 1.

[14] M. Nakajima, H. Negoro, R. Sasaki et al., MAXI/GSC observation of the giant outburst from the Be/X-ray binary pulsar A 0535+262, The Astronomer's Telegram 14173 (2020) 1.

[15] J. van den Eijnden, N. Degenaar, R. Wijnands, T.D. Russell, G.R. Sivakoff, J.C.A. Miller-Jones et al., $V L A$ detection of the radio counterpart of the BeXRB 1A 0535+262, The Astronomer's Telegram 14193 (2020) 1.

[16] S. Treuz, V. Doroshenko, A. Santangelo and R. Staubert, Distances to Accreting X-ray pulsars: impact of the Gaia DR2, arXiv e-prints (2018) arXiv:1806.11397 [1806.11397].

[17] W.C. Priedhorsky and J. Terrell, 111-day periodicity of X-ray transient A0535 + 26, Nature 303 (1983) 681.

[18] G.K. Jaisawal, J.B. Coley, J. Wilms et al., Super-critical accretion onto the Be/X-ray binary pulsar $1 A$ 0535+262 during its 2020 giant X-ray outburst, The Astronomer's Telegram 14227 (2020) 1.

[19] J. Aleksić, S. Ansoldi, L.A. Antonelli, P. Antoranz, A. Babic, P. Bangale et al., The major upgrade of the MAGIC telescopes, Part I: The hardware improvements and the commissioning of the system, Astroparticle Physics 72 (2016) 61 [1409.6073].

[20] J. Aleksić, S. Ansoldi, L.A. Antonelli, P. Antoranz, A. Babic, P. Bangale et al., The major upgrade of the MAGIC telescopes, Part II: A performance study using observations of the Crab Nebula, Astroparticle Physics 72 (2016) 76 [1409.5594].

[21] J. Aleksić, E.A. Alvarez, L.A. Antonelli, P. Antoranz, M. Asensio, M. Backes et al., Detection of VHE $\gamma$-Rays from HESS J0632+057 during the 2011 February X-Ray Outburst with the MAGIC Telescopes, ApJL 754 (2012) L10 [1203 . 2867].

[22] J.S. Bright, R.P. Fender, S.E. Motta, D.R.A. Williams, J. Moldon, R.M. Plotkin et al., An extremely powerful long-lived superluminal ejection from the black hole MAXI J1820+070, Nature Astronomy 4 (2020) 697 [2003.01083].

[23] J. Colomé, I. Ribas, X. Francisco, K. Casteels, D. Fernández, J. Isern et al., The OAdM Robotic Observatory, Advances in Astronomy 2010 (2010) 183016.

[24] C. Celma, The bright outburst of a new black hole candidate: Optical observations of MAXI J1820+070 from the Montsec observatory, Master's thesis, Universitat Politècnica de Catalunya, 2019.

[25] T.P. Li and Y.Q. Ma, Analysis methods for results in gamma-ray astronomy., ApJ 272 (1983) 317.

\section{The MAGIC Collaboration}

V. A. Acciari ${ }^{1}$, S. Ansoldi ${ }^{2,41}$, L. A. Antonelli ${ }^{3}$, A. Arbet Engels ${ }^{4}$, M. Artero ${ }^{5}$, K. Asano ${ }^{6}$, D. Baack ${ }^{7}$, A. Babié ${ }^{8}$, A. Baquero ${ }^{9}$, U. Barres de Almeida ${ }^{10}$, J. A. Barrio ${ }^{9}$, I. Batković ${ }^{11}$, J. Becerra González ${ }^{1}$, W. Bednarek ${ }^{12}$, L. Bellizzi ${ }^{13}$, E. Bernardini ${ }^{14}$, M. Bernardos ${ }^{11}$, A. Berti ${ }^{15}$, J. Besenrieder ${ }^{15}$, W. Bhattacharyya ${ }^{14}$, C. Bigongiari ${ }^{3}$, A. Biland ${ }^{4}$, O. Blanch ${ }^{5}$, H. Bökenkamp ${ }^{7}$, G. Bonnoli ${ }^{16}$, Ž. Bošnjak $^{8}$, G. Busetto ${ }^{11}$, R. Carosi ${ }^{17}$, G. Ceribella ${ }^{15}$, M. Cerruti ${ }^{18}$, Y. Chai ${ }^{15}$, A. Chilingarian ${ }^{19}$, S. Cikota ${ }^{8}$, S. M. Colak ${ }^{5}$, E. Colombo ${ }^{1}$, J. L. Contreras ${ }^{9}$, J. Cortina ${ }^{20}$, S. Covino ${ }^{3}$, G. D’Amico ${ }^{15,42}$, V. D’Elia ${ }^{3}$, P. Da Vela ${ }^{17,43}$, F. Dazzi ${ }^{3}$, A. De Angelis ${ }^{11}$, B. De Lotto ${ }^{2}$, M. Delfino ${ }^{5,44}$, J. Delgado ${ }^{5,44}$, C. Delgado Mendez $^{20}$, D. Depaoli ${ }^{21}$, F. Di Pierro ${ }^{21}$, L. Di Venere ${ }^{22}$, E. Do Souto Espiñeira ${ }^{5}$, D. Dominis Prester $^{23}$, A. Donini ${ }^{2}$, D. Dorner ${ }^{24}$, M. Doro ${ }^{11}$, D. Elsaesser ${ }^{7}$, V. Fallah Ramazani ${ }^{25,45}$, A. Fattorini ${ }^{7}$, M. V. Fonseca ${ }^{9}$, L. Font ${ }^{26}$, C. Fruck ${ }^{15}$, S. Fukami ${ }^{6}$, Y. Fukazawa ${ }^{27}$, R. J. García López ${ }^{1}$, M. Garczarczyk ${ }^{14}$, S. Gasparyan ${ }^{28}$, M. Gaug ${ }^{26}$, N. Giglietto ${ }^{22}$, 
F. Giordano ${ }^{22}$, P. Gliwny ${ }^{12}$, N. Godinović ${ }^{29}$, J. G. Green ${ }^{3}$, D. Green ${ }^{15}$, D. Hadasch ${ }^{6}$, A. Hahn ${ }^{15}$, L. Heckmann ${ }^{15}$, J. Herrera ${ }^{1}$, J. Hoang ${ }^{9,46}$, D. Hrupec ${ }^{30}$, M. Hütten ${ }^{15}$, T. Inada ${ }^{6}$, K. Ishio ${ }^{12}$, Y. Iwamura ${ }^{6}$, I. Jiménez Martínez ${ }^{20}$, J. Jormanainenn ${ }^{25}$, L. Jouvin ${ }^{5}$, M. Karjalainen ${ }^{1}$, D. Kerszberg ${ }^{5}$, Y. Kobayashi' ${ }^{6}$, H. Kubo ${ }^{31}$, J. Kushida ${ }^{32}$, A. Lamastra ${ }^{3}$, D. Lelas ${ }^{29}$, F. Leone ${ }^{3}$, E. Lindfors ${ }^{25}$, L. Linhoff ${ }^{7}$, S. Lombardi ${ }^{3}$, F. Longo ${ }^{2,47}$, R. López-Coto ${ }^{11}$, M. López-Moya ${ }^{9}$, A. López-Oramas ${ }^{1}$, S. Loporchio ${ }^{22}$, B. Machado de Oliveira Fraga $^{10}$, C. Maggio ${ }^{26}$, P. Majumdar ${ }^{33}$, M. Makariev ${ }^{34}$, M. Mallamaci ${ }^{11}$, G. Maneva ${ }^{34}$, M. Manganaro ${ }^{23}$, K. Mannheim ${ }^{24}$, L. Maraschi ${ }^{3}$, M. Mariotti ${ }^{11}$, M. Martínez ${ }^{5}$, D. Mazin ${ }^{6,15}$, S. Menchiari ${ }^{13}$, S. Mender ${ }^{7}$, S. Mićanović23, D. Miceli ${ }^{2,49}$, T. Miener ${ }^{9}$, J. M. Miranda ${ }^{13}$, R. Mirzoyan ${ }^{15}$, E. Molina ${ }^{18}$, A. Moralejo ${ }^{5}$, D. Morcuende ${ }^{9}$, V. Moreno ${ }^{26}$, E. Moretti ${ }^{5}$, T. Nakamori ${ }^{35}$, L. Nava ${ }^{3}$,

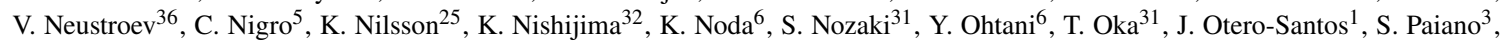
M. Palatiello ${ }^{2}$, D. Paneque ${ }^{15}$, R. Paoletti ${ }^{13}$, J. M. Paredes ${ }^{18}$, L. Pavletić $^{23}$, P. Peñil ${ }^{9}$, M. Persic ${ }^{2,50}$, M. Pihet ${ }^{15}$, P. G. Prada Moroni ${ }^{17}$, E. Prandini ${ }^{11}$, C. Priyadarshi ${ }^{5}$, I. Puljak ${ }^{29}$, W. Rhode ${ }^{7}$, M. Ribó ${ }^{18}$, J. Rico ${ }^{5}$, C. Righi ${ }^{3}$, A. Rugliancich ${ }^{17}$, N. Sahakyan ${ }^{28}$, T. Saito ${ }^{6}$, S. Sakurai ${ }^{6}$, K. Satalecka ${ }^{14}$, F. G. Saturni ${ }^{3}$, B. Schleicher ${ }^{24}$, K. Schmidt ${ }^{7}$, T. Schweizer ${ }^{15}$, J. Sitarek ${ }^{12}$, I. Šnidaric ${ }^{37}$, D. Sobczynska ${ }^{12}$, A. Spolon ${ }^{11}$, A. Stamerra ${ }^{3}$, J. Strišković ${ }^{30}$, D. Strom ${ }^{15}$, M. Strzys ${ }^{6}$, Y. Suda ${ }^{27}$, T. Surić ${ }^{37}$, M. Takahashi ${ }^{6}$, R. Takeishi ${ }^{6}$, F. Tavecchio ${ }^{3}$, P. Temnikov ${ }^{34}$, T. Terzić ${ }^{23}$, M. Teshima ${ }^{15,6}$, L. Tosti ${ }^{38}$, S. Truzzi $^{13}$, A. Tutone ${ }^{3}$, S. Ubach ${ }^{26}$, J. van Scherpenberg ${ }^{15}$, G. Vanzo $^{1}$, M. Vazquez Acosta ${ }^{1}$, S. Ventura ${ }^{13}$, V. Verguilov ${ }^{34}$, C. F. Vigorito ${ }^{21}$, V. Vitale ${ }^{39}$, I. Vovk $^{6}$, M. Will ${ }^{15}$, C. Wunderlich ${ }^{13}$, T. Yamamoto $^{40}$, and D. Zaric ${ }^{29}$

${ }^{1}$ Instituto de Astrofísica de Canarias and Dpto. de Astrofísica, Universidad de La Laguna, E-38200, La Laguna, Tenerife, Spain ${ }^{2}$ Università di Udine and INFN Trieste, I-33100 Udine, Italy ${ }^{3}$ National Institute for Astrophysics (INAF), I-00136 Rome, Italy ${ }^{4}$ ETH Zürich, CH-8093 Zürich, Switzerland ${ }^{5}$ Institut de Física d'Altes Energies (IFAE), The Barcelona Institute of Science and Technology (BIST), E-08193 Bellaterra (Barcelona), Spain ${ }^{6}$ Japanese MAGIC Group: Institute for Cosmic Ray Research (ICRR), The University of Tokyo, Kashiwa, 277-8582 Chiba, Japan ${ }^{7}$ Technische Universität Dortmund, D-44221 Dortmund, Germany ${ }^{8}$ Croatian MAGIC Group: University of Zagreb, Faculty of Electrical Engineering and Computing (FER), 10000 Zagreb, Croatia ${ }^{9}$ IPARCOS Institute and EMFTEL Department, Universidad Complutense de Madrid, E-28040 Madrid, Spain ${ }^{10}$ Centro Brasileiro de Pesquisas Físicas (CBPF), 22290-180 URCA, Rio de Janeiro (RJ), Brazil ${ }^{11}$ Università di Padova and INFN, I-35131 Padova, Italy ${ }^{12}$ University of Lodz, Faculty of Physics and Applied Informatics, Department of Astrophysics, 90-236 Lodz, Poland ${ }^{13}$ Università di Siena and INFN Pisa, I-53100 Siena, Italy ${ }^{14}$ Deutsches Elektronen-Synchrotron (DESY), D-15738 Zeuthen, Germany ${ }^{15}$ Max-Planck-Institut für Physik, D-80805 München, Germany ${ }^{16}$ Instituto de Astrofísica de Andalucía-CSIC, Glorieta de la Astronomía s/n, 18008, Granada, Spain ${ }^{17}$ Università di Pisa and INFN Pisa, I-56126 Pisa, Italy ${ }^{18}$ Universitat de Barcelona, ICCUB, IEEC-UB, E-08028 Barcelona, Spain ${ }^{19}$ Armenian MAGIC Group: A. Alikhanyan National Science Laboratory, 0036 Yerevan, Armenia ${ }^{20}$ Centro de Investigaciones Energéticas, Medioambientales y Tecnológicas, E-28040 Madrid, Spain ${ }^{21}$ INFN MAGIC Group: INFN Sezione di Torino and Università degli Studi di Torino, I-10125 Torino, Italy 22 INFN MAGIC Group: INFN Sezione di Bari and Dipartimento Interateneo di Fisica dell'Università e del Politecnico di Bari, I-70125 Bari, Italy ${ }^{23}$ Croatian MAGIC Group: University of Rijeka, Department of Physics, 51000 Rijeka, Croatia ${ }^{24}$ Universität Würzburg, D-97074 Würzburg, Germany ${ }^{25}$ Finnish MAGIC Group: Finnish Centre for Astronomy with ESO, University of Turku, FI-20014 Turku, Finland ${ }^{26}$ Departament de Física, and CERES-IEEC, Universitat Autònoma de Barcelona, E-08193 Bellaterra, Spain 27 Japanese MAGIC Group: Physics Program, Graduate School of Advanced Science and Engineering, Hiroshima University, 739-8526 Hiroshima, Japan ${ }^{28}$ Armenian MAGIC Group: ICRANet-Armenia at NAS RA, 0019 Yerevan, Armenia ${ }^{29}$ Croatian MAGIC Group: University of Split, Faculty of Electrical Engineering, Mechanical Engineering and Naval Architecture (FESB), 21000 Split, Croatia ${ }^{30}$ Croatian MAGIC Group: Josip Juraj Strossmayer University of Osijek, Department of Physics, 31000 Osijek, Croatia ${ }^{31}$ Japanese MAGIC Group: Department of Physics, Kyoto University, 606-8502 Kyoto, Japan ${ }^{32}$ Japanese MAGIC Group: Department of Physics, Tokai University, Hiratsuka, 259-1292 Kanagawa, Japan ${ }^{33}$ Saha Institute of Nuclear Physics, HBNI, 1/AF Bidhannagar, Salt Lake, Sector-1, Kolkata 700064, India ${ }^{34}$ Inst. for Nucl. Research and Nucl. Energy, Bulgarian Academy of Sciences, BG-1784 Sofia, Bulgaria ${ }^{35}$ Japanese MAGIC Group: Department of Physics, Yamagata University, Yamagata 990-8560, Japan ${ }^{36}$ Finnish MAGIC Group: Astronomy Research Unit, University of Oulu, FI-90014 Oulu, Finland ${ }^{37}$ Croatian MAGIC Group: Ruđer Bošković Institute, 10000 Zagreb, Croatia ${ }^{38}$ INFN MAGIC Group: INFN Sezione di Perugia, I-06123 Perugia, Italy ${ }^{39}$ INFN MAGIC Group: INFN Roma Tor Vergata, I-00133 Roma, Italy ${ }^{40}$ Japanese MAGIC Group: Department of Physics, Konan University, Kobe, Hyogo 6588501, Japan ${ }^{41}$ also at International Center for Relativistic Astrophysics (ICRA), Rome, Italy ${ }^{42}$ now at Department for Physics and Technology, University of Bergen, NO-5020, Norway ${ }^{43}$ now at University of Innsbruck ${ }^{44}$ also at Port d'Informació Científica (PIC), E-08193 Bellaterra (Barcelona), Spain ${ }^{45}$ now at Ruhr-Universität Bochum, Fakultät für Physik und Astronomie, Astronomisches Institut (AIRUB), 44801 Bochum, Germany ${ }^{46}$ now at Department of Astronomy, University of California Berkeley, Berkeley CA 9472047 also at Dipartimento di Fisica, Università di Trieste, I-34127 Trieste, Italy 49 now at Laboratoire d'Annecy de Physique des Particules (LAPP), CNRS-IN2P3, 74941 Annecy Cedex, France ${ }^{50}$ also at INAF Trieste and Dept. of Physics and Astronomy, University of Bologna, Bologna, Italy 